\title{
Politique
}

Politique

\section{Monique Dupuis, Crise mondiale et aide internationale; Stratégie canadienne et développement du Tiers-Monde, Montréal, Nouvelle Optique, 1984, 182 p.}

\section{Sylvain Ayotte}

Numéro 7, hiver 1985

Projection internationale du Québec

URI : https://id.erudit.org/iderudit/040486ar

DOI : https://doi.org/10.7202/040486ar

Aller au sommaire du numéro

Éditeur(s)

Société québécoise de science politique

ISSN

0711-608X (imprimé)

1918-6584 (numérique)

Découvrir la revue

Citer ce compte rendu

Ayotte, S. (1985). Compte rendu de [Monique Dupuis, Crise mondiale et aide internationale; Stratégie canadienne et développement du Tiers-Monde, Montréal, Nouvelle Optique, 1984, 182 p.] Politique, (7), 128-133.

https://doi.org/10.7202/040486ar d'utilisation que vous pouvez consulter en ligne.

https://apropos.erudit.org/fr/usagers/politique-dutilisation/ 
Monique Dupuis, Crise mondiale et aide internationale; Stratégie canadienne et développement du Tiers-Monde, Montréal, Nouvelle Optique, $1984,182 \mathrm{p}$.

Depuis ses débuts, l'aide canadienne a toujours occupé une place importante au chapitre de la politique étrangère du Canada. À ce titre, étant donné que les études consacrées exclusivement ou en partie à l'aide canadienne se font relativement rares, l'ouvrage de Monique Dupuis s'avère d'un intérêt certain.

Le constat que fait l'auteure en début d'analyse est que les milliards fournis par le biais de l'aide publique au développement (APD) n'ont pas réduit les inégalités entre pays riches et pauvres, pas plus qu'ils n'ont empêché l'élargissement du fossé entre les mieux nantis et les plus démunis des sociétés du Tiers-Monde. On se propose donc d'expliquer pourquoi l'ADP se maintient et se développe si son but n'est pas de réduire la misère. (p. 9).

Afin de saisir la problématique de l'aide, il sera nécessaire de la replacer dans son contexte: de nos jours, celle-ci n'est plus nationale, mais se situe au niveau de l'évolution de la structure mondiale. De là la nécessité de mettre en relation la stratégie canadienne d'aide au développement avec une stratégie de restructuration du système mondial.

La thèse, avancée par Monique Dupuis, est à l'effet que la stratégie canadienne d'aide s'inscrit harmonieusement dans le processus de restructuration du système mondial, mis de l'avant par les principaux stratèges (identifiés comme l'avant-garde capitaliste) que sont la Commission Trilatérale, la Banque Mondiale, la Commission Brandt, etc. Cela étant dit, il ne faudrait pas penser que dans la perspective de l'auteure les intérêts du capital transnational vont à l'encontre des intérêts canadiens, mais plutôt que ceux-ci y trouvent leur compte parce qu'ils sont tributaires du capital transnational, en partie sous hégémonie américaine. 
L'étude, dont l'objet global est le rôle de la coopération internationale, se veut en quelque sorte une analyse de la stratégie canadienne d'aide au développement mise en rapport spécifiquement avec les objectifs "homogènes" qu'entretiennent la Commission Trilatérale et la Commission Brandt au sujet de la restructuration du système mondial. À cet effet, l'ouvrage est divisé en deux parties traitant, d'une part, de la nature du système mondial tel qu'aiguillé par l'avant-garde capitaliste et, part, de l'aide publique au développement et de la stratégie canadienne d'aide.

Dans la première tranche de l'étude, l'auteur adopte l'approche du système de l'économie mondiale (SEM) développée par Charles A. Michalet. Cette approche postule que l'unité d'analyse à privilégier est le phénomène de l'internationalisation de la production, caractérisé par la délocalisation de la production industrielle et l'émergence de structures transnationales. Dans cette optique, la mondialisation du mode de production capitaliste, qui a entraîné la mondialisation de la lutte des classes, a provoqué un blocage de l'accumulation, donc du point de vue de l'auteure, la crise en elle-même (p. 20).

Selon l'analyse de Monique Dupuis, la Commission Trilatérale préconise que la restructuration du système mondial doit être orientée par une restructuration économique (accentuation de l'internationalisation du capital et de la division internationale du travail) et une restructuration politique (concertation des impérialismes occidentaux, encerclement et à plus long terme inclusion du bloc de l'est dans le SEM, consolidation des alliances avec les bourgeoisies périphériques et limitation de la démocratie), dont la légitimation idéologique est chapeautée par les concepts clés de la sécurité nationale et l'interdépendance des économies. Le but de cette restructuration consiste à maintenir une stabilité politique et économique à long terme de l'ordre mondial fournissant les conditions optimales de développement du capital transnational. 
Après un examen relativement rapide du rapport de la Commission Brandt sur les problèmes de développement international, l'auteure affirme que celui-ci s'inscrit parfaitement dans le cadre circonscrit par la Commission Trilatérale. Deux arguments interreliés lui permettent de prendre une telle position. Dans un premier temps, on évalue que la Commission Brandt a volontairement évacué la dimension historique du problème du développement, au profit de la «célèbre» cause du retard de la croissance économique et de l'industrialisation (pp. 60-61). Dans un second temps, la stratégie, qui est liée à la problématique identifiée, fait largement référence à l'industrialisation (p. 64), et en conséquence on y indique qu'en cette matière l'Occident et son capital peuvent y jouer un rôle de premier plan. Pour l'auteure, une telle stratégie se ferait nécessairement au détriment de l'agriculture et du développement autocentré sur un plan plus général.

Dans son introduction à la seconde partie du volume, Monique Dupuis a bien pris soin de brosser un tableau synthèse de l'aide publique au développement. Le rôle de l'aide y est notamment perçu comme l'instrument des puissances impérialistes ayant pour but de redéfinir le lien colonial (p. 77). En dressant un bilan de l'aide canadienne, l'auteure déplore que seulement $6 \%$, en moyenne, des fonds de l'APD du Canada soient affectés aux organismes nongouvernementaux, organismes pourtant reconnus pour la qualité et le caractère bénéfique de leurs travaux. De même, on insiste beaucoup sur le fait que le Canada, malgré qu'il se situe au premier rang pour la libéralité des conditions relatives aux prêts, est l'un des plus conservateurs au chapitre de l'aide liée. La conséquence du liement de l'aide canadienne est que celle-ci se voit confinée à des projets à haute technologie et intensité de capital, qui mettent généralement l'accent sur le transfert des capitaux pour l'infrastructure (p. 101).

Par ailleurs, l'auteure dénote qu'au fil des ans à l'Agence canadienne de développement international (ACDI), même s'il y 
a eu des changements d'orientation, les objectifs sont demeurés invariables, à savoir que l'aide est conçue avant tout comme un instrument de politique étrangère et que celle-ci est conditionnée par de multiples intérêts nationaux. La performance canadienne en matière d'aide s'explique principalement, pour Monique Dupuis, par les fondements théoriques et idéologiques qui prévalent à l'Agence canadienne de développement international et au Ministère des Affaires Extérieures. La théorie qui semble avoir primé est celle des «étapes de la croissance économique» de W.W. Rostow, selon laquelle les États du Tiers-Monde ne sont qu'en retard ou dans une phase primaire du processus de développement, d'où la pertinence de «collaborer» à la hausse de la croissance économique par le biais de l'apport industriel.

En bref, selon Monique Dupuis, le Canada est un État impérialiste de puissance moyenne et impérialisé par les ÉtatsUnis. De par son passé, le Canada qui n'avait jamais eu d'aspirations coloniales se devait d'appuyer la décolonisation et, par le biais de l'aide, encourager la promotion du modèle occidental de la démocratie libérale et du capitalisme. Ainsi l'image favorable que s'est attiré le Canada lui permettra d'occuper le rôle de remplaçant des puissances impériales auxquelles il est le plus lié. Actuellement, l'État canadien voit ses intérêts nationaux bien servis par l'internationalisation de son capital, ce qui dans un cadre plus global contribue fortement à la restructuration du système mondial, telle que préconisée par l'avant-garde capitaliste.

L'intérêt premier de cet ouvrage est certes l'objet d'analyse comme tel: les études approfondies concernant l'aide canadienne étant rarissismes, l'accessibilité à l'information dans ce domaine y est par surcroît relativement difficile, étant donné son éparpillement et dans certains cas sa confidentialité, ce qui s'avère tout à l'honneur de l'auteure.

Par contre, bien que l'idée d'évaluer la stratégie de l'aide canadienne à la lumière du processus de restructuration du système 
mondial nous est apparue fort intéressante; il semble que l'auteure ait glissé dans le piège analytique que constitue la Commission Trilatérale en tant qu'objet d'étude. Il faut bien reconnaitre d'une part que, tout aussi prestigieux et influents que soient les membres de ce club sélect, la Trilatérale n'est pas aussi homogène que l'on «voudrait bien le croire». Il faut comprendre, d'autre part, que cette Commission n'est que la manifestation organisationnelle du capital transnational, en quelque sorte la pointe de l'iceberg. Dans ce contexte, il ne nous apparaît pas indubitable que la restructuration du système mondial n'aurait pas suivi son cours sans la contribution de la Commission Trilatérale. Ainsi, l'analyse de la stratégie canadienne d'aide au développement perd de son attrait dans la mesure où celle-ci n'est plus en référence à un processus de restructuration du système mondial, mais strictement fonction des discours et intérêts de quelques élites hautement influentes du capital transnational. En sens inverse, il nous aurait semblé plus pertinent, sur le plan de l'analyse, que l'auteure approfondisse par exemple, davantage qu'elle ne l'a fait, l'approche du système de l'économie mondiale de C.A. Michalet, ce qui aurait certainement eu pour effet d'apporter une meilleure compréhension de la structure mondiale.

Nous avons cependant perçu d'un point de vue plus positif la tentative formulée par l'auteure pour démontrer que dans les institutions vouées au développement international prévaut une réflexion théorique bien précise du développement, à savoir celle de W.W. Rostow exposée dans Les étapes de la croissance économique. À ce titre, nous croyons qu'il aurait été profitable que Monique Dupuis développe plus longuement la critique de ce modèle, ce qui aurait pu lui permettre d'étoffer d'une manière plus explicite sa conception d'une alternative au développement, puisqu'elle lui consacre moins de deux pages en fin d'analyse.

En bref, il s'agit d'un ouvrage intéressant pour la synthèse des informations relatives aux questions de développement et à 
la stratégie canadienne d'aide, dont la portée analytique se révèle toutefois minée par une trop grande importance accordée à l'aspect visuel du capital transnational.

Sylvain Ayotte

Université Laval 